Address correspondence to: Fitz-Roy E. Curry, Department of Physiology and Membrane Biology, School of Medicine, One Shields Avenue, University of California Davis, Davis, California 95616, USA. Phone: (530) 752-7081; Fax: (530) 7525423; E-mail: fecurry@ucdavis.edu.

1. Michel, C.C., and Curry, F.E. 1999. Microvascular permeability. Physiol. Rev. 79:703-761.
2. Brenner, B.M., Ballermann, B.J., Gunning, M.E., and Zeidel, M.L. 1990. Diverse biological actions of atrial natriuretic peptide. Physiol. Rev. 70:665-699.

3. Baxter, G.F. 2004. The natriuretic peptides. Basic Res. Cardiol. 99:71-75

4. Tucker, V.L. 1996. Plasma ANP levels and protein extravasation during graded expansion with equilibrated whole blood. Am. J. Physiol. 271:R601-R609.

5. Renkin, E.M., and Tucker, V.L. 1996. Atrial natriuretic peptide as a regulator of transvascular fluid balance. News Physiol. Sci. 11:138-143.

6. Sabrane, K., et al. 2005. Vascular endothelium is critically involved in the hypotensive and hypovolemic actions of atrial natriuretic peptide. J. Clin. Invest. 115:1666-1674. doi:10.1172/JCI23360.

7. Holtwick, R., et al. 2002. Smooth muscle-selective deletion of guanylyl cyclase-A prevents the acute but not chronic effects of ANP on blood pressure. Proc. Natl. Acad. Sci. U. S. A. 99:7142-7147.

8. Adamson R.H., et al. 2004. Oncotic pressures opposing filtration across non-fenestrated rat microvessels. J. Physiol. 557:889-907.

\title{
Lessons learned from cancer may help in the treatment of pulmonary hypertension
}

\section{Serge Adnot}

INSERM U651 and Département de Physiologie, Hôpital H. Mondor, Assistance Publique des Hôpitaux de Paris, Créteil, France.

\begin{abstract}
Hyperplasia of pulmonary artery SMCs (PASMCs) is a pathological hallmark of pulmonary arterial hypertension (PAH). In this issue of the JCI, McMurtry et al. report that adenovirus-mediated overexpression of survivin - a multipotent inhibitor of apoptosis - induces PAH in rats, whereas inhalation of an adenovirus vector encoding a mutant survivin gene with dominantnegative properties reverses established monocrotaline-induced PAH (see the related article beginning on page 1479). These findings raise important issues regarding the role of survivin in the pathogenesis of $\mathrm{PAH}$, its value as a prognostic indicator, and its use as a target for new therapeutic strategies.
\end{abstract}

\section{Pulmonary arterial hypertension and smooth muscle hyperplasia: unresolved questions}

Pulmonary arterial hypertension (PAH), whether idiopathic (iPAH) or associated with underlying diseases (aPAH), is an unexplained condition for which severe forms in adults or neonates are fatal and no satisfactory treatment is available. Valuable new insights into the disease have stemmed in recent years from genetic studies identifying mutations in the gene encoding bone morphogenic protein receptor type 2 (BMPRII) in patients with familial iPAH $(1,2)$ and from functional studies showing dysregulation of molecular events governing pulmonary artery SMC (PASMC) proliferation (3). The nature of the primary defect responsible for triggering, maintaining, and arresting PASMC

Nonstandard abbreviations used: aPAH, PAH associated with underlying diseases; BMP, bone morphogenic protein; BMPRII, bone morphogenic protein receptor type 2; ET-1, endothelin-1; 5-HHT, serotonin transporter; 5-HT, serotonin; iPAH, idiopathic PAH; PAH, pulmonary arterial hypertension; PASMC, pulmonary artery SMC.

Conflict of interest: The author has declared that no conflict of interest exists.

Citation for this article: J. Clin. Invest. 115:1461-1463 (2005). doi:10.1172/JCI25399. proliferation in PAH is poorly understood but may be either an inherent characteristic of PASMCs (as seen with cancer cells) or a secondary response to an external abnormality, such as upregulation of a growth factor. Under the first hypothesis, cells removed from their in vivo environment would continue to proliferate excessively, whereas under the second hypothesis, their proliferation rate would be normal. An intermediate situation has been found: when removed from their in vivo environment, PASMCs from PAH patients demonstrate excessive proliferation when stimulated by serotonin (5-hydroxytryptamine [5-HT]) or endothelin-1 (ET-1) but not when stimulated by PDGF, EGF, FGF, IGF, or TGF- $\beta(4,5)$. Moreover, they are far less sensitive to growth inhibition by bone morphogenic proteins (BMPs) than are normal cells (6). Therefore, the intrinsic abnormalities of these PASMCs involve not intracellular mitogenic signals but rather signal transmission from specific autocrine or paracrine factors such as BMPs, 5-HT, or ET-1. Increased expression of the serotonin transporter (5-HTT), which mediates the proliferative effect of 5-HT, and marked reduction of BMPRII expression appear specific to $\mathrm{PAH}$ and
aPAH $(4,7)$. These molecular alterations occur without apparent genetic abnormalities: in cells without the mutation in BMPRII, the extent of BMPRII underexpression is similar to that in mutated cells (6), and the 5-HTT overexpression can be explained only partly by the polymorphism of its gene promoter (5). Why PASMCs from $\mathrm{PAH}$ patients maintain an abnormal phenotype in vitro and how environmental and genetic factors regulate the expression of these key molecular transmitters remain open questions.

Until now, alterations in apoptotic processes have not been considered to play a role in PASMC proliferation, in contrast to mechanisms of disorganized EC proliferation contributing to plexiform lesions at branch points of pulmonary arteries in iPAH (8). In this issue of the JCI, McMurtry et al. open up the intriguing possibility that increased survivin expression may contribute to the abnormal PASMC phenotype observed in PAH (9).

\section{Is there a role for survivin} in the pathogenesis of PAH?

Survivin belongs to the inhibitor of apoptosis protein (IAP) family, members of which confer protection from deathinducing stimuli (10). One of the most striking features of survivin is its differential expression in cancerous versus normal tissues. Survivin is virtually undetectable in normal adult differentiated tissues but is expressed in most human cancers (11). Thus, dysregulated survivin expression is considered to be a major pathologic mechanism of apoptosis inhibition. However, survivin pathway reactivation may occur 


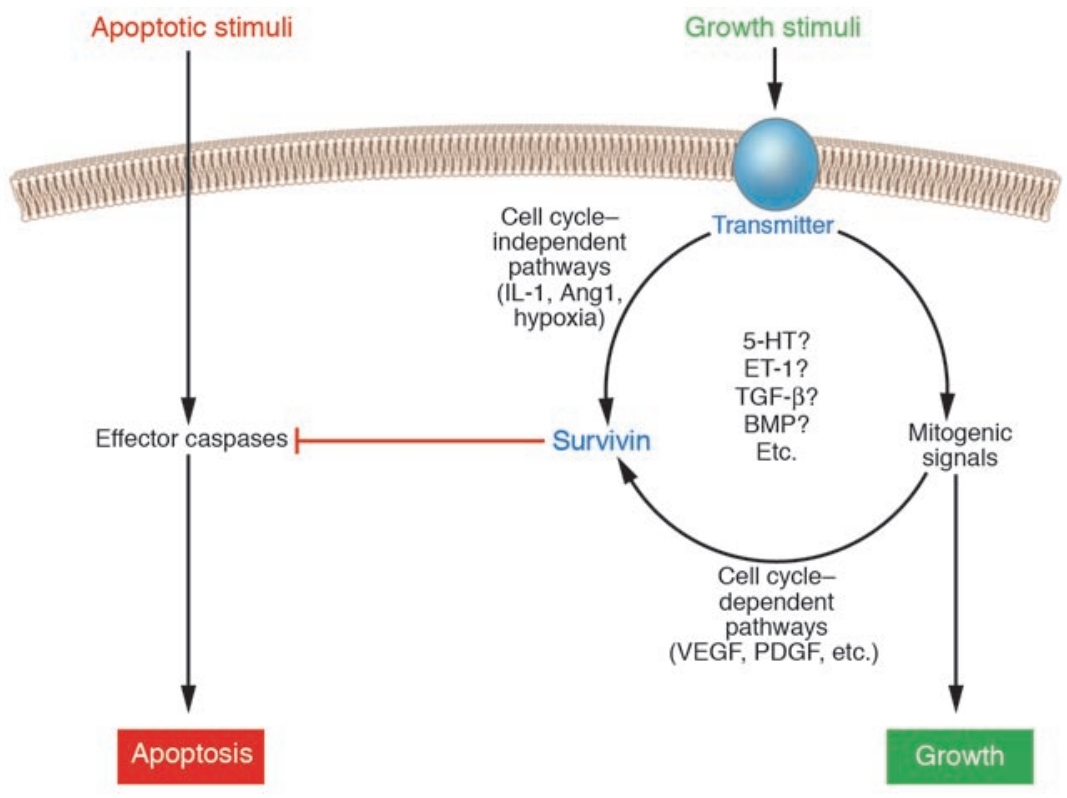

Figure 1

Hypothetical mechanisms leading to hyperplasia of SMCs or ECs in $\mathrm{PAH}$. A number of growth stimuli may act predominantly by inducing mitogenic signals, which may then lead to survivin expression through cell cycle-dependent pathways (cells stimulated by VEGF, PDGF, etc.). Stimuli with a predominant antiapoptotic effect may act on survivin expression chiefly through cell cycle-independent pathways (cells stimulated by IL-1, angiopoietin-1 [Ang1], hypoxia, etc.). The potential effects of factors involved in the pathogenesis of PAH, such as 5-HT, ET-1, TGF- $\beta$, and BMPs are unknown.

in normal cell types, most notably vascular cells, including SMCs and ECs. That SMC survival influences abnormal vessel wall remodeling is supported by data from studies of systemic arteries. Vascular injury increases survivin expression in the vessel wall, concomitantly with neointima formation (12). Similarly, McMurtry and colleagues demonstrate that survivin overexpression coincided with pulmonary vascular remodeling in rats developing monocrotaline-induced PAH (9). Thus, survivin overexpression does not precede, but instead occurs downstream of, the remodeling process. Interestingly, in vitro stimulation of SMCs from systemic arteries also induces survivin expression, in keeping with the fact that survivin is regulated in a cell cycle-dependent manner (10). However, in SMCs from systemic arteries, this occurs in response to PDGF but not to EGF, basic FGF, or TGF- $\beta$ (12). On the other hand, ECs express survivin in response to angiogenic factors that stimulate growth, such as VEGF, but also in response to angiopoietin-1, which on the contrary induces EC quiescence while increasing EC survival (13, 14). Thus, vascular cell stimuli may induce survivin expression, mitogenic signals, or a com- bination of both (Figure 1). The response may depend on the type of stimulus but may also vary according to the origin and phenotype of the vascular cells. Whether dysregulated survivin expression is associated with the abnormal PASMC phenotype in PAH and whether it is affected selectively by specific growth factors are important questions for future research.

A major finding from the study by McMurtry et al. (9) is that lung gene transfer of survivin triggers pulmonary vascular remodeling. Antiapoptotic signals and growth factors are believed to act together to produce PASMC proliferation in PAH. The fact that a selective increase in an antiapoptotic signal induces PAH indicates that regulation of the balance between mitotic and apoptotic activity in the normal pulmonary circulation is critical to maintaining pulmonary vessel integrity. Since survivin gene transfer to cultured SMCs does not cause cell growth, this suggests that, in vivo, growth stimuli are continuously delivered to the normal pulmonary vessels. Thus, among stimuli causing PAH, some may act only by stimulating survivin. This may be particularly relevant to disease states, since survivin expression is not only regulated in a cell cycle-dependent man- ner but is also under the influence of many factors, including hypoxia and ubiquitous transcription factors such as NF-KB (10).

\section{Is survivin a valuable prognostic index in pulmonary hypertension?}

In several tumor series, presence of survivin correlated with a lower apoptotic index in vivo and shorter overall patient survival (15, 16). Whether these characteristics apply to iPAH is of great interest. There is considerable dissimilarity among patients with PAH regarding disease progression rates and treatment responses. Whether the level of survivin expression in pulmonary vessels reflects the potential for disease progression remains to be tested.

\section{Survivin antagonists: a novel therapeutic strategy for pulmonary hypertension?}

The use of molecular antagonists of survivin to increase cell death might hold therapeutic potential, most notably for tumors (11). Because survivin is expressed chiefly in growing tissues, limited toxicity for normal tissues would be expected. Previous in vivo results published in the JCI are consistent with this possibility, showing selective inhibition of tumor growth in mice treated with a survivin mutant adenovirus (17). Survivin antagonists hold appeal in the treatment of PAH, as they may work in the various forms of PAH, despite differences in pathogenic mechanisms. However, the view that survivin is expressed only in cancers has been challenged by many studies. Survivin expression has been documented in adult quiescent cells, including several cell types in the central nervous system, circulating neutrophils and lymphocytes, hematopoietic stem cells, hepatocytes, and intestinal crypt cells $(11,13,18)$. Administration of survivin antagonists via an intratracheal route may limit untoward systemic effects but may not be devoid of toxicity to airways and alveolar epithelial cells or to ECs. Thus, whether survivin antagonists can be safely administered to $\mathrm{PAH}$ patients without affecting systemic organs and lung cell types other than PASMCs will have to be extensively studied before these agents are considered for therapeutic purposes.

Address correspondence to: Serge Adnot, INSERM U651, Département de Physiologie, Faculté de Médecine de Créteil, 94010 Créteil, France. Phone: 33-1-49-81-3693, Fax: 33-1-48-98-17-77; E-mail: serge. adnot@hmn.ap-hop-paris.fr. 
1. Deng, Z., et al. 2000. Familial primary pulmonary hypertension (gene PPH1) is caused by mutations in the bone morphogenetic protein receptor-II gene. Am. J. Hum. Genet. 67:737-744.

2. Lane, K., et al. 2000. Heterozygous germline mutations in BMPR2, encoding a TGF-beta receptor, cause familial primary pulmonary hypertension. The International PPH Consortium. Nat. Genet. 26:3-4.

3. Eddahibi, S., Morrell, N., d'Ortho, M., Naeije, R., and Adnot, S. 2002. Pathobiology of pulmonary arterial hypertension. Eur. Respir. J. 20:1559-1572.

4. Eddahibi, S., et al. 2001. Serotonin transporter overexpression is responsible for pulmonary artery smooth muscle hyperplasia in primary pulmonary hypertension. J. Clin. Invest. 108:1141-1150. doi:10.1172/JCI200112805.

5. Marcos, E., et al. 2004. Serotonin-induced smooth muscle hyperplasia in various forms of human pulmonary hypertension. Circ. Res. 94:1263-1270.

6. Morrell, N.W., et al. 2001. Altered growth responses of pulmonary artery smooth muscle cells from patients with primary pulmonary hypertension to transforming growth factor-beta(1) and bone morphogenetic proteins. Circulation. 104:790-795.

7. Atkinson, C., et al. 2002. Primary pulmonary hypertension is associated with reduced pulmonary vascular expression of type II bone morphogenetic protein receptor. Circulation. 105:1672-1678.

8. Lee, S.D., et al. 1998. Monoclonal endothelial cell proliferation is present in primary but not secondary pulmonary hypertension. J. Clin. Invest. 101:927-934.

9. McMurtry, M.S., et al. 2005. Gene therapy targeting survivin selectively induces pulmonary vascular apoptosis and reverses pulmonary arterial hypertension. J. Clin. Invest. 115:1479-1491. doi:10.1172/ JCI23203.

10. Salvesen, G.S., and Duckett, C.S. 2002. IAP proteins: blocking the road to death's door. Nat. Rev. Mol. Cell Biol. 3:401-410.

11. Altieri, D.C. 2003. Validating survivin as a cancer therapeutic target. Nat. Rev. Cancer. 3:46-54.

12. Blanc-Brude, O.P., et al. 2002. Inhibitor of apoptosis protein survivin regulates vascular injury. Nat. Med. 8:987-994.
13. Conway, E.M., et al. 2003. Survivin-dependent angiogenesis in ischemic brain: molecular mechanisms of hypoxia-induced up-regulation. Am. J. Pathol. 163:935-946.

14. Tran, J., et al. 2002. A role for survivin in chemoresistance of endothelial cells mediated by VEGF. Proc. Natl. Acad. Sci. U. S. A. 99:4349-4354.

15. Kato, J., et al. 2001. Expression of survivin in esophageal cancer: correlation with the prognosis and response to chemotherapy. Int. J. Cancer. 95:92-95.

16. Monzo, M., et al. 1999. A novel anti-apoptosis gene: re-expression of survivin messenger RNA as a prognosis marker in non-small-cell lung cancers. J. Clin. Oncol. 17:2100-2104.

17. Mesri, M., Wall, N.R., Li, J., Kim, R.W., and Altieri, D.C. 2001. Cancer gene therapy using a survivin mutant adenovirus. J. Clin. Invest. 108:981-990. doi:10.1172/JCI200112983.

18. Wang, K., Brems, J., Gamelli, R., and Ding, J. 2005. Reversibility of caspase activation and its role during glycochenodeoxycholate-induced hepatocyte apoptosis. J. Biol. Chem. doi:10.1074/jbc. M411607200.

\title{
Stem cell-ness: a "magic marker" for cancer
}

\author{
John P. Lahad,1 Gordon B. Mills, ${ }^{1}$ and Kevin R. Coombes²
}

${ }^{1}$ Department of Molecular Therapeutics and 2Department of Biostatistics and Applied Mathematics, University of Texas M.D. Anderson Cancer Center, Houston, Texas, USA.

\begin{abstract}
Transcriptional profiling of patient tumors is a much-heralded advancement in cancer therapy, as it provides the opportunity to identify patients who would benefit from more or less aggressive therapy and thus allows the development of individualized treatment. However, translation of this promise into patient benefit has proven challenging. In this issue of the $J C I$, Glinsky and colleagues used human and murine models to identify a potential stem cell mRNA signature, based on the hypothesis that tumors with stem cell-like characteristics are likely to have a poor prognosis (see the related article beginning on page 1503). Remarkably, an 11-gene "expression signature" associated with "stem cell-ness" separated patients with different cancers into good-and poor-prognosis groups. Such a "magic marker" would, if validated, have a major impact on patient care. However, there remain challenges incumbent with creating and validating such signatures.
\end{abstract}

\section{Prediction and cancer}

The inception of high-throughput analyses using oligonucleotide microarrays has given biologists the ability to globally assess RNA levels in a patient's tumor sample. A

Nonstandard abbreviations used: $A N K 3$, ankyrin 3; Bmi-1, murine leukemia viral-1; BUB1, budding uninhibited by benzimidazoles 1 ; CCNB1, cyclin B1; CES, carboxylesterase; FGFR2, FGF receptor 2; GBX2, gastrulation homeobox 2; HCFC1, host cell factor c1; Ki-67, antigen identified by mAb ki-67; KNTC2, kinetochore-associated 2; PNS, peripheral nervous system; RNF2, ring finger 2; SPAI, stem cell-like phenotype association index; TRAMP, transgenic adenocarcinoma of the mouse prostate; USP22, ubiquitin-specific protease 22.

Conflict of interest: The authors have declared that no conflict of interest exists.

Citation for this article: J. Clin. Invest. 115:1463-1467 (2005). doi:10.1172/JCI25455. typical microarray study generates data on the expression of the approximately 20,000 human genes and soon studies will be able to analyze the more than 150,000 splice variants of RNA that are likely to have functional roles. The inherent challenge is to convert this data into applicable knowledge. A potential strength of the technology lies in its ability to uncover complex gene interaction patterns and correlate those patterns with clinically relevant outcomes. This "holy grail" could ultimately predict not only the therapeutic response of the tumor present in each patient, but also the patient's survival, which would subsequently lead to the development of individualized therapy for each patient based both on the genetic aberrations in the tumor and on the patient's own genetic makeup. However, despite early enthusiasm, there have been considerable challenges in converting the promise of individualized molecular medicine into clinical practice.

In this issue of the JCI, Glinsky and colleagues outline a possible expression signature comprising 11 genes that has the ability, according to the authors' analysis, to segregate tumor samples from multiple tumor lineages into those that have good or poor prognoses (1). The authors have applied this gene set to multiple tissue types from disparate data sets and have repeatedly observed its predictive power. The application of their 11-gene signature to these independent sets addresses an analytical limitation that is often overlooked when "predictive" gene expression signatures are found in microarray experiments $(2,3)$. When thousands of measurements are taken on each patient, the number of ways to select some of those measurements as a pattern classifying tumors or predicting outcomes is enormous. When selecting multiple gene measurements, the probability of finding a combination with apparent clinical relevance just by chance is even higher.

This multiple-measurements problem can be addressed using a "training and test set" approach, wherein predictive models are validated on separate, independent 